\title{
Correlation between malnutrition, body mass index and complications in patients with urinary bladder cancer who underwent radical cystectomy
}

\author{
Marta Swalarz ${ }^{1, A-D}$, Grzegorz Swalarz 2,B,D, Kajetan Juszczak ${ }^{3, B, C, E, F}$, Piotr Maciukiewicz ${ }^{3, B},{ }^{2}$, Krzysztof Czurak ${ }^{4, B}$, \\ Marcin Matuszewski, ${ }^{4, B}$, Dominika Gajewska ${ }^{5, B}$, Marcin Słojewski, ${ }^{5, B}$, Rafał Bogacki ${ }^{6, B}$, Piotr Bryniarski ${ }^{6, A-C, E, F}$, \\ Andrzej Paradysz ${ }^{6, A, B}$, Mateusz Kadłubowski ${ }^{7}, B$, Tomasz Drewa ${ }^{7, A, B}$, Ewa Genge ${ }^{8, C, E, F}$ \\ ${ }^{1}$ Silesian Center of UROVITA, Chorzów, Poland \\ 2 Department of Surgery and Urology, Upper Silesian Child Health Center, Katowice, Poland \\ ${ }^{3}$ Department of Urology, Memorial Rydygier Hospital, Kraków, Poland \\ ${ }^{4}$ Department of Urology, University Clinical Centre, Gdańsk, Poland \\ ${ }^{5}$ Department of Urology and Urological Oncology, Pomeranian Medical University, Szczecin, Poland \\ ${ }^{6}$ Department of Urology, Medical University of Silesia, Zabrze, Poland \\ ${ }^{7}$ Division of General and Oncological Urology, Specialized Municipal Hospital, Toruń, Poland \\ ${ }^{8}$ Department of Economic and Financial Analysis, University of Economics, Katowice, Poland \\ A - research concept and design; B - collection and/or assembly of data; C - data analysis and interpretation; \\ $D$ - writing the article; $E$ - critical revision of the article; $F$ - final approval of the article
}

Address for correspondence

Marta Swalarz

E-mail:martusiaswalarz@gmail.com

Funding sources

None declared

Conflict of interest

None declared

\section{Acknowledgements}

Special thanks to PhD Andrzej Szurkowski

for the opportunity to conduct the research

and to all professors and doctors involved

in this project.

Received on August 23, 2016

Reviewed on March 5, 2017

Accepted on March 14, 2018

DOI

10.17219/acem/89863

Copyright

Copyright by Author(s)

This is an article distributed under the terms of the

Creative Commons Attribution Non-Commercial License

(http://creativecommons.org/licenses/by-nc-nd/4.0/)

\section{Abstract}

Background. Nutrition is the $3^{\text {rd }}$ most important factor in surgery, following anesthesia and asepsis. Until now, it has been a poorly explored field of urology. The relationship between malnutrition and postoperative complications has been proven beyond doubt in general surgery, where $30 \%$ of patients are operated in a malnutrition state.

Objectives. The aim of our work was to assess the influence of malnutrition, defined by nutritional risk screening (NRS) scale and body mass index (BMI), on postoperative results in patients with bladder cancer after radical cystectomy.

Material and methods. The research was carried out at 8 urological centers between 2012 and 2014, and included patients with bladder cancer at stage from T2 to T4, who underwent radical cystectomy. The degree of malnutrition was assessed with the aid of the NRS 2002 questionnaire. Other examined parameters were BMI, age, type of operation, and the number of complications, the latter of which were measured by applying the Clavien-Dindo scale.

Results. A total of 125 patients were enrolled in our study, out of whom 64 (51.2\%) were undernourished. According to the BMI, most of the patients were overweight - 50 (40\%) or had normal body weight - 49 (39.2\%); 24 (19.2\%) were obese, and 2 (1.6\%) were underweight.

Conclusions. There was no relationship between malnutrition, defined by the NRS scale, and postoperative complications, and we did not find a significant relationship between the other tested variables. We observed only 1 significant relationship between the nutrition state, measured by BMI scale, and the degree in Clavien-Dindo scale. Body mass index under 18.5 and over 30 increased postoperative complications. Nowadays, the recommended scale is NRS 2002, which is based mostly on loss of weight. In our patients, qualitative malnutrition is more probable than quantitative malnutrition.

Key words: malnutrition, bladder cancer, postoperative complications, radical cystectomy, Clavien-Dindo scale 


\section{Introduction}

Nutrition is the $3^{\text {rd }}$ most important factor in surgery, following anesthesia and asepsis. The World Health Organization (WHO) defines malnutrition as "occurring at the cellular imbalance between the demand for nutrients and energy supply and demand, which can satisfy the growth with, vital functions and perform certain functions". Basically, incorrect nutrition occurs when the body does not receive enough energy (quantitative malnutrition - weight loss) or essential nutrients, such as protein, vitamins, minerals and other nutrients needed to maintain healthy tissues and organs (quantitative malnutrition - e.g., vitamin deficiency). Both kinds of malnutrition can naturally coexist in the same patient. Such a condition is not limited only to patients who are obviously malnourished; it can also affect overweight and obese people. Malnutrition results from little consumption or not digesting or absorbing essential nutrients with regard to the needs of the body, or from the excretion of these nutrients faster than they can be completed. Loss of nutrients may be accelerated by a wide variety of factors, including surgery, diarrhea, severe intestinal dysfunctions, burns, sweating, severe bleeding, or as in our case, hematuria, renal failure or cancer. Malnutrition in hospitalized patients is a common phenomenon and its consequences are underestimated. According to reports, about 35-55\% of patients admitted to hospital are malnourished and this situation has not changed since the early 1970s. ${ }^{1,2}$

Undernutrition affects the structure and function of certain cells, tissues and organs. We used to define malnutrition by dysfunctions of organs, when undernutrition already changes particular biochemical parameters (serum albumin level, lymphocytes level, etc.). But dysfunction of organs is not the best indicator of malnutrition, because it's malnutrition occurs much earlier. Research was undertaken to establish classification systems that can recognize disturbances in nutritional state faster, so that the improper trend of nutrition could be quickly reversed, thus preventing the dysfunction of cells.

In our paper we used the Nutritional Risk Screening 2002 (NRS 2002) scale, recommended by the European Society for Clinical Nutrition and Metabolism (ESPEN), Polish Society of Parenteral Nutrition, Enteral Nutrition and Metabolism (POLSPEN), and National Insurance Organization (NFZ), to detect and define undernutrition. This scale was also chosen to describe malnutrition due to its international popularity and usefulness. In Poland, each adult patient admitted to hospital should undergo a nutritional assessment test using NRS or SGA (Subjective Global Assessment) scale. The purpose was to detect the presence of undernutrition and also to detect patients at risk of developing undernutrition in hospital conditions. Chosen parameters show the moment when prevention steps should be taken to avoid function deteriorations, worsening of postoperative complications and prolonged recovery time, which result in increased hospitalization costs.
The latest research, describes the impairment of function as a result of various extents of weight loss, with various rates of weight loss from various initial nutritional statures, on the basis of these studies, the NRS scale was created and is widespread. Controlled trials again found that such an approach has excellent inter-rater reliability, concurrent validity with other tools, and predictive validity (length of hospital stay, mortality in elderly wards and discharge destination in orthopedic patients). These optimistic scores have convinced us to check if by assessing the nutritional state we can really predict the probability of postoperative complication severity in urological patients undergoing radical cystectomy (RC). These patients suffer from many complications. That is why we are still looking for factors which could decrease the number of complications. One of these factors could be poor preoperative nutritional status, which is a proven risk factor for adverse outcomes after major surgery.

Malnutrition does not affect only people who are underweight. Increasingly more often it concerns overweight or obese people, whose diet consists of consuming high amounts of calories, mostly from carbohydrates, which are low in vitamins and minerals. The number of people worldwide who are overweight or obese is still growing and nowadays amounts to 2.1 billion. In Poland it constitutes $53 \%$ of the population; moreover, if we consider people over 45 years of age, it amounts to $77 \%$ of the population. The average body mass index (BMI) in Poland is 25.9. The relationship between $\mathrm{BMI}$ and cancer has been proven, which also applies to bladder cancer $(\mathrm{BCa}){ }^{3}$

Obesity is a risk factor responsible for an increased number of postoperative complications in surgery and orthopedics. ${ }^{4,5}$ Bladder cancer ranks $4^{\text {th }}$ in men and $8^{\text {th }}$ in women, as far as incidence of all malignant tumors in Poland is considered. Annually, there are 5,700 new cases, out of which $25 \%$ are in $\mathrm{T} 2-\mathrm{T} 4$ stage. $\mathrm{RC}$ remains a gold standard treatment for muscle-invasive $\mathrm{BCa}$ and plays a role in non-muscle-invasive disease. About 2/3 of patients suffer from 1 or more complications, such as excessive loss of blood, urinary leakage, venous thromboembolic disease, digestive tract symptoms, ureteral reflux, acute renal failure, etc. ${ }^{6,7}$

The relationship between malnutrition and postoperative complications has been proven beyond doubt in general surgery. Until now, it has been a poorly explored area in the field of urology. Therefore, we decided to evaluate the correlation between malnutrition, BMI and complications in patients with urinary bladder cancer who underwent RC.

\section{Material and methods}

A multicenter study aimed at examining the state of malnutrition in patients who had undergone $\mathrm{RC}$, was carried out at 8 urological centers (21 patients from hospitals in Toruń, 20 from a hospital in Gdańsk, 20 from 
Szczecin, 3 from Kraków, and 61 from 4 Silesian hospitals - Chorzów, Zabrze, Bielsko-Biała, and Katowice) between 2012 and 2014. The study was not a medical experiment, which explains why approval from the bioethics committee was not necessary. A total of 125 patients (mean age 65.2; median 64) were examined and our study group consisted of $14 \%$ women and $86 \%$ men. All patients who were prepared to RC were screened by NRS 2002 to estimate nutritional status. According to that scale, patients were asked to give their weight from 3 months before, and we measured their body mass prior to surgery. The percentage of body mass loss was calculated, and also other variables given in the NRS 2002 questionnaire were taken into consideration, such as the following: BMI, food intake, age, and severity of disease. According to ESPEN guidelines, all patients who received a score $\geq 3$ were considered patients nutritionally at risk who should have a nutritional care plan prepared. Patients with a score over 2 in the NRS scale were considered nutritionally deficient (ND) patients. Only patients who had undergone RC because of muscle-invasive BC (in T2-T4 stage) were admitted to this research, while $\mathrm{RC}$ due to other reasons was an exclusion criterion. Due to the fact that it was the same disease and the same stage of the disease (T2-T4), all patients got 2 points in NRS scale for the severity of disease.

The variable in our study was nutritional state (NS), which is a binary variable: NND - not nutritionally deficient (patients with score under 3), and ND - nutritionally deficient (patients with score over 2). The other variable was the degree of malnutrition (DM), depending on the number of points, which had 4 categories: $\mathrm{NN}$ - normal nutrition; DM1 $-1^{\text {st }}$ degree of malnutrition, which constituted patients who got $3(1+2)$ points in the NRS scale, i.e., those whose loss of weight amounted to $5-10 \%$ of body mass within last 3 months, whose food intake was between $50-75 \%$ of normal requirement in preceding week, or who were over 70 years of age; DM2 - constituted patients who got $4(2+2)$ points in NRS scale, i.e., those who were in the $2^{\text {nd }}$ degree of malnutrition, whose loss of weight was more than $10 \%$ of body mass within last 3 months, whose food intake, was between $25-60 \%$ of normal requirement in the preceding week or their BMI indicates undernutrition, so was between $18.5-20.5$ or was in the $1^{\text {st }}$ degree of malnutrition because of weight loss or food intake and who were over 70 years of age; DM3 - patients in the $3^{\text {rd }}$ degree of malnutrition, who got $5(3+2)$ points in NRS scale, i.e., those whose loss of weight was over $15 \%$ of body mass within last 3 months, whose food intake was below $25 \%$ of normal requirement in the preceding week, whose BMI was under 18.5 or was in the $2^{\text {nd }}$ degree of malnutrition because of weight loss or food intake, and who were over 70 years of age. All patients from the ND group were asked to give causes for their loss of body mass.

Aside from our patients being classified according to the NRS scale, their BMI was also calculated, and the patients were divided into categories according to the BMI scale: BMI1 - underweight patients with a BMI under 18.5; BMI2 patients with normal body weight and BMI from 18.5 up to 25; BMI3 overweight patients with a BMI from 25 up to 30; and BMI4 - obese patients with a BMI over 30. Moreover, out of ND variables, we selected patients with a BMI over 25 and we created an additional group, which consisted of obese or overweight patients who were at the same time undernourished, due to big body mass loss within last 3 months. Other examined parameters were the type of operation and postoperative complications measured in the Clavien-Dindo scale. If the patient had above 2 points in the Clavien-Dindo scale, we considered it as clinically important. The total duration of the study was approx. 2 years. We must admit that in some hospitals parenteral nutrition and blood infusion (when hemoglobin level is $<8$ ) after RC were the standard procedure of postoperative care.

\section{Statistical analysis}

The $\chi^{2}$ test and the Fisher's exact test (for contingency tables with small counts) for independence were used to assess the relationships between categorical variables and the eta correlation coefficient was used as a correlation indicator for variables measured on mixed scales. Correspondence analysis was applied as a statistical visualization method for picturing the associations between the levels of a 2-way contingency table. SPPS and R statistical software packages (SPSS Inc., Chicago, USA) were used in our computations.

\section{Results}

Out of 125 patients (mean age 65.2: median 64), 61 (48.8\%) were not nutritionally deficient (NND) and 64 (51.2\%) were nutritionally deficient (ND) (Table 1). Regarding the degree of malnutrition, most patients were in the DM1 category - 29 (23.2\%), DM2 constituted 20 (16\%) patients and DM3 constituted 15 (12\%) (Table 2). The main cause of malnutrition was mostly the disease itself (chronic hematuria and pain, which resulted in the loss of appetite); other causes was stress before the surgery, deliberately going on a diet, or undergoing neoadjuvant chemotherapy, which lowered patients' appetite and resulted in weight loss. According to the BMI scale, BMI3 and BMI2 constituted almost the same number of patients: BMI3 - $50(40 \%)$, BMI2 - 49 (39.2\%) and BMI4 - 24 (19.2\%). This suggested that $54.4 \%$ of our patients were overweight or obese, which is much lower than in the Polish population, where the percentage of people over 45 years of age with a BMI over 25 amounts to $77 \%$, BMI1-2 (1.6\%). The group of obese and overweight patients, who had become malnourished before surgery due to big body mass loss, constituted $36(28.8 \%)$ patients. 
Table 1. Contingency table for "nutritional status" and complications in Clavien-Dindo scale variables

\begin{tabular}{|l|c|c|}
\hline $\begin{array}{c}\text { Score in Clavien- } \\
\text { Dindo scale/ } \\
\text { nutritional status }\end{array}$ & NND & ND \\
\hline 1 & $19(15.2 \%)$ & $15(12 \%)$ \\
\hline 2 & $36(28.8 \%)$ & $37(29.6 \%)$ \\
\hline 3 & $2(1.6 \%)$ & $4(3.2 \%)$ \\
\hline 5 & $3(2.4 \%)$ & $4(3.2 \%)$ \\
\hline
\end{tabular}

NND - not nutritionally deficient; ND - nutritionally deficient.

The number of clinically important complications over 2 in the Clavien-Dindo scale in NS variables was 18 (14.4\%) of patients (Table 1) and was slightly higher in categories of ND patients - $12(9.6 \%)(4[3.2 \%]+4[3.2 \%]+4[3.2 \%])$ to $6(4.8 \%)(2[1.6 \%]+3[3.2 \%]+1[0.8 \%])$ in NND patients. The degree in the Clavien-Dindo scale compared to the degree of malnutrition variable showed that the group of patients, in which serious complications were most likely to occur, was DM2, where 25\% (5 out of 20) of patients had complications above 2, while in the DM1 group it was $17.2 \%$ (5 out of 29 ) and in DM3 - 13\% (2 out of 15); in the NN group serious complications could be observed only in $9.8 \%$ (6 out of 61 ) of patients (Table 2). The rate of complications according to the BMI scale is presented in Table 3. The most popular urinary diversion was the Bricker method, performed in 87 (69.6\%) patients, while ureterocutaneostomy was performed in 31 (24.8\%) patients and ileal neobladder reconstruction by the Student's t-test was performed in 7 (5.6\%) patients (Table 4).

\section{Results of statistical analysis}

Pearson's $X^{2}$ test of independence was used to check whether a statistically significant relationship between variables existed. Due to the small sample sizes (cell size below 5 expected occurrences), the Fisher's exact test for contingency tables with small samples was used. The frequency distribution of the variables was analyzed on the basis of cross tabulations, which provided a basic picture of the interrelation between variables. Most patients who had clinical, but not serious complications, were nutritionally normal. The results of the Pearson's $\chi^{2}$ and the Fisher's exact tests between different pairs of variables, as well as eta correlation coefficient, are given in Table 5 . In the conducted tests, only 1 significant relationship between nutrition state, measured by BMI, and the degree in Clavien-Dindo scale $(\mathrm{p}=0.0274)$ was observed. Therefore, the null hypothesis could not be rejected and a significant relationship between those variables was observed.

Correspondence analysis was applied as an exploratory data analytic technique to present relationships among the categories of those variables (BMI and complications). In Fig. 1, the proximity of the $4^{\text {th }}$ degree of complication
Table 2. Contingency table for degree of malnutrition (DM) and complications in Clavien-Dindo scale variables

\begin{tabular}{|l|c|c|c|c|}
\hline $\begin{array}{c}\text { Score } \\
\text { in Clavien-Dindo } \\
\text { scale }\end{array}$ & NN & DM1 & DM2 & DM3 \\
\hline 1 & $19(15.2 \%)$ & $7(5.6 \%)$ & $3(2.4 \%)$ & $5(4 \%)$ \\
\hline 2 & $36(28.8 \%)$ & $17(13.6 \%)$ & $12(9.6 \%)$ & $8(6.4 \%)$ \\
\hline 3 & $2(1.6 \%)$ & $2(1.6 \%)$ & $1(0.8 \%)$ & $1(0.8 \%)$ \\
\hline 4 & $3(2.4 \%)$ & 0 & $3(2.4 \%)$ & $1(0.8 \%)$ \\
\hline 5 & $1(0.8 \%)$ & $3(2.4 \%)$ & $1(0.8 \%)$ & 0 \\
\hline
\end{tabular}

NN - nutritionally normal; DM1 - $1^{\text {st }}$ degree of malnutrition;

$\mathrm{DM} 2-2^{\text {nd }}$ degree of malnutrition; DM3 - $3^{\text {rd }}$ degree of malnutrition.

Table 3. Number of complications in Clavien-Dindo scale in nutritional groups according to the body mass index (BMI) scale

\begin{tabular}{|l|l|l|l|l|}
$\begin{array}{c}\text { Score } \\
\text { in Clavien-Dindo } \\
\text { scale }\end{array}$ & BMI1 & BMI2 & BMI3 & BMI4 \\
\hline 1 & 0 & $16(12.8 \%)$ & $15(12 \%)$ & $3(2.4 \%)$ \\
\hline 2 & $1(0.8 \%)$ & $22(17.6 \%)$ & $30(24 \%)$ & $20(16 \%)$ \\
\hline 3 & $1(0.8 \%)$ & $5(4 \%)$ & 0 & 0 \\
\hline 4 & 0 & $4(3.2 \%)$ & $2(1.6 \%)$ & $1(0.8 \%)$ \\
\hline 5 & 0 & $2(1.6 \%)$ & $3(2.4 \%)$ & 0 \\
\hline
\end{tabular}

BMI1 - under 18.5; BMI2 - 18.5-24.9; BMI3 - 25-29.9; BMI4 - above 30.

Table 4. Number of complications in Clavien-Dindo scale in patients with different urinary diversions

\begin{tabular}{|l|c|c|c|}
$\begin{array}{c}\text { Score } \\
\text { in Clavien-Dindo } \\
\text { scale }\end{array}$ & Bricker & Studer & Ureterocutaneostomy \\
\hline 1 & $21(16.8 \%)$ & $4(3.2 \%)$ & $9(7.2 \%)$ \\
\hline 2 & $53(42.4 \%)$ & $2(1.6 \%)$ & $18(14.4 \%)$ \\
\hline 3 & $4(3.2 \%)$ & 0 & $2(1.6 \%)$ \\
\hline 4 & $7(5.6 \%)$ & 0 & 0 \\
\hline 5 & $2(1.6 \%)$ & $1(0.8 \%)$ & $2(1.6 \%)$ \\
\hline
\end{tabular}

Table 5. Measures of relationships and significance levels

\begin{tabular}{|c|c|c|c|}
\hline \multirow[b]{2}{*}{ Relationship } & \multirow[b]{2}{*}{ Statistic } & \multicolumn{2}{|c|}{$\mathrm{p}$-value } \\
\hline & & $x^{2}$ & $\begin{array}{l}\text { Fisher's } \\
\text { exact test }\end{array}$ \\
\hline NS-complications & $x^{2}=3.6743$ & 0.4519 & 0.4740 \\
\hline DM-complications & $x^{2}=11.7798$ & 0.4635 & 0.3952 \\
\hline BMI-complications & $x^{2}=24.8512$ & 0.0155 & 0.0274 \\
\hline Bricker-complications & $x^{2}=6.4500$ & 0.1680 & 0.1406 \\
\hline Studer-complications & $x^{2}=6.3064$ & 0.1774 & 0.1473 \\
\hline $\begin{array}{l}\text { Ureterocutaneostomy- } \\
\text { complications }\end{array}$ & $x^{2}=2.6720$ & 0.6141 & 0.6389 \\
\hline Bricker-DM & $x^{2}=2.3417$ & 0.5046 & 0.5266 \\
\hline Studer-DM & $x^{2}=2.9466$ & 0.3999 & 0.5782 \\
\hline Ureterocutaneostomy-DM & $x^{2}=5.4272$ & 0.1431 & 0.1557 \\
\hline Age-complications & $\eta=0.423$ & 0.1830 & - \\
\hline
\end{tabular}

$D M$ - degree of malnutrition; $x^{2}-$ Pearson's $x^{2}$ test. 
to the circle representing the $1^{\text {st }}$ category of $\mathrm{BMI}$ indicates that the $4^{\text {th }}$ degree in Clavien-Dindo scale is associated with the underweight patients. The association between patients with normal body weight (BMI2) and overweight patients (BMI3) with the $2^{\text {nd }}$ degree of complications can be noticed as well.

It might seem difficult to find associations between categories of the variables, which is why Ward's method was used; it is one of the best classification methods to interpret the perception map of the correspondence analysis. The categories were clustered on the basis of calculated coordinates. As a result of clustering analysis, a dendrogram was created using the $\mathrm{R}$ statistical package (Fig. 2). On the basis of Silhoutte index (the measure of cluster separation), 3 clusters, which describe the relationship between examined categories, were selected. The $1^{\text {st }}$ cluster consisted of obese patients associated with $3^{\text {rd }}$ degree in Clavien-Dindo scale. In the $2^{\text {nd }}$ cluster, the association between patients with normal body weight and overweight with the $2^{\text {nd }}$ degree of complications can be observed. In the $3^{\text {rd }}$ cluster, the underweight patients are strongly associated with the $4^{\text {th }}$ degree in the Clavien-Dindo scale and weakly associated with the $1^{\text {st }}$ and $5^{\text {th }}$ category of complications.

\section{Discussion}

In our study, more complications were found in the ND group, but no statistical relationship between those variables was noticed. ${ }^{5,8-12}$ It is possible that increasing the number of patients in the study group could lead us to different conclusions. We should remember that malnutrition, defined by the NRS scale, constitutes patients not only in an undernutrition state, but also patients at risk of malnutrition, i.e., the status in which patients are in an imbalance between the amount of absorbed and used substances. This creates disturbances in the structure and function of cells and organs. The main problem in urological patients is chronic hematuria causing a loss of a lot of nutritional substances, which may induce malnutrition without a decrease in weight.

Therefore, further prospective studies are needed to more precisely determine the usefulness of measuring nutritional status by the NRS scale. Age and the selected type of urinary diversion also did not increase the amount of complications in a significant way. ${ }^{9,12}$ A relationship between complications and BMI was the only significant relationship that we could notice also in other studies. ${ }^{3,7,13,14}$ In the group of ND overweight or obesity, no increased number of complications was noticed either. Based on those facts, it should be further examined if losing mass in the case of patients with BMI over 25 is a protective factor. Of course, losing mass will never be a goal for patients with cancer. However, it requires further studies to make sure if putting our patients on a healthy norm-caloric diet will help them to limit the number of complications.

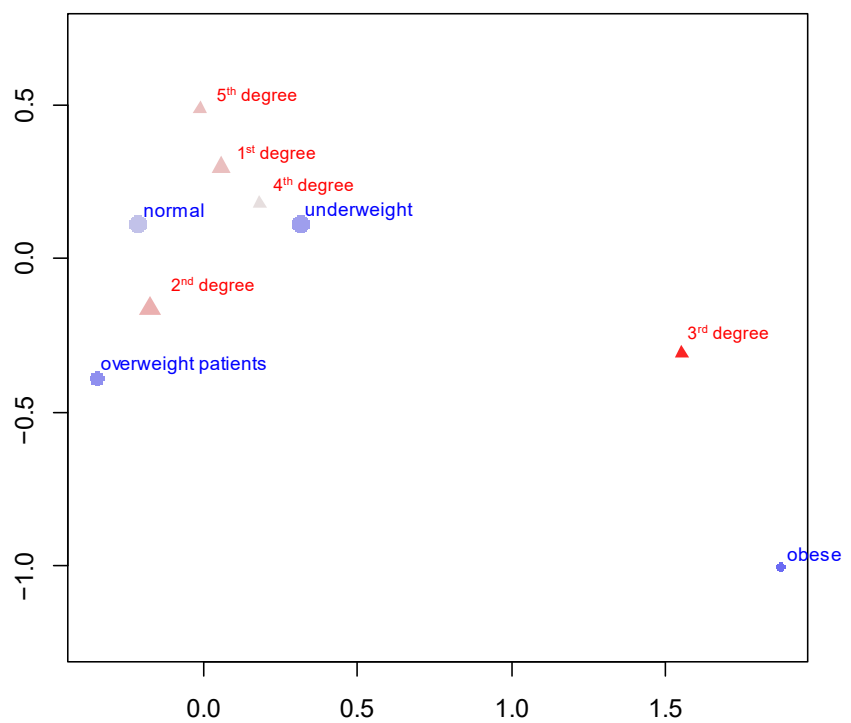

Fig. 1. Correspondence analysis plot

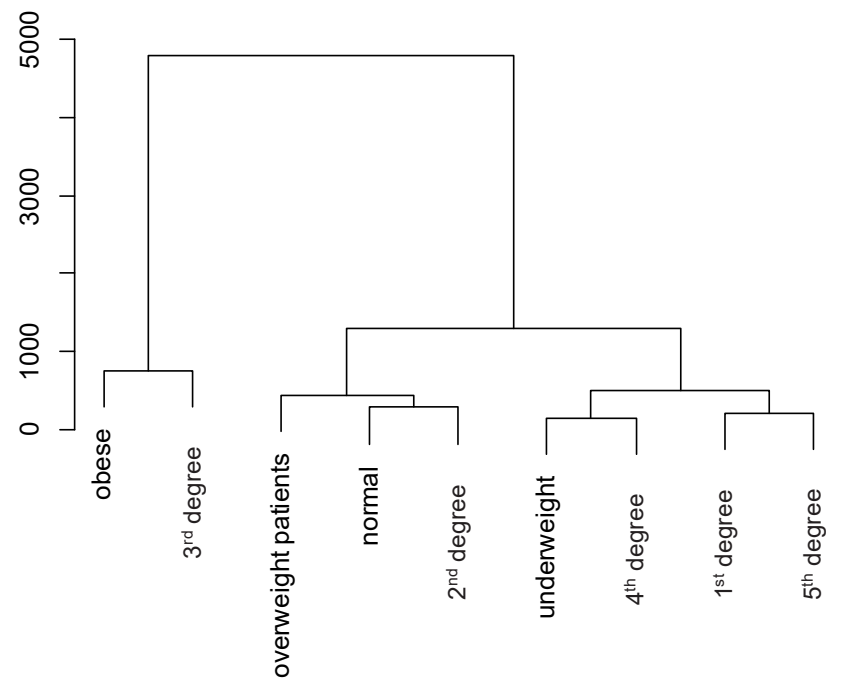

Fig. 2. Dendrogram for different categories of variables: complications in Clavien-Dindo scale and body mass index (BMI)

Many reports show which vitamins and minerals are in deficiency in our population; it is also well known which microelements are necessary for wounds to heal properly. However, this knowledge is not utilized well enough before important and cost-generating procedures like RC. The patients who undergo $\mathrm{RC}$, besides cancer, suffer from a lot of other diseases; therefore, their condition should be optimized before the operation. Seriously ill patients should be provided with a nutritional, physical and mental health program as a standard procedure. An American survey conducted in 2014, entitled "Optimizing a frail elderly patient for radical cystectomy with a prehabilitation program", proposes and shows a holistic preparation program, including nutritional counseling, protein supplementation, anxiety reduction, and a moderate exercise program. ${ }^{15}$ This prehabilitation program was also checked 
in patients after colorectal surgery. ${ }^{16}$ An individualized approach and precise preparation of patients proved to increase recovery in walking capacity, as well as emotional and cognitive functions, and also decreased the number of postoperative complications.

In order to improve the nutritional status in an optimized way, we should have reliable information which patients are in need of special nutritional support. Good nutritional markers or scales, which show the real nutritional problems of patients, are of primary importance.

According to the classification of NRS 2002 scale, in our study, $51.2 \%$ of patients were undernutritioned, which is a high number. In contrast to general surgery, undernutrition classified in such a way does not influence postoperative complications. The most frequent cause of losing weight was the disease itself. The possible explanation of this fact is that due to chronic hematuria, the urological patient is more likely to suffer from qualitative rather than from quantitative malnutrition. Consequently, a patient without weight loss may be malnourished. Therefore, the scale NRS 2002 does not seem to be adequate, as it does not reflect the real condition of the nutritional state of our patients, who, despite no weight loss, may have serious element deficits, e.g., iron and other minerals, which are absolutely necessary for proper healing of wounds after operation. It is possible that measuring other biochemical indices, e.g., erythrocyte count, hemoglobin concentration, American Society of Anesthesiologists (ASA) classification score over 3 , ferritin serum concentration, C-reactive protein (CRP) neutrophil-to-lymphocyte ratio, as well as the level of antioxidant vitamins (A, C, E, D), vitamin $\mathrm{B}_{12}$ and folic acid, will be useful for more reliable diagnosing of underfed patients who need nutritional support. ${ }^{6,17-24}$ Serum albumin is omitted in the new nutritional scales, which is why during planning this survey we did not take this parameter into consideration. Some research works say that "albumin and other visceral protein should no longer be considered as nutritional markers, but as inflammatory response markers", while others claim that "serum levels of albumin, prealbumin, transferrin and IGF-1, and delayed hypersensitivity and total lymphocyte count may be valid to help stratify risk. However, it is not appropriate to consider these as markers of adequacy of nourishment in the sick patient."13,14 A number of composite measures of nutritional status were proposed, although no standardized method of nutrition evaluation exists, especially for urological patients. ${ }^{12} \mathrm{Nu}$ tritional Risk Screening 2002 is nowadays a validated index, but it is based on subjective information, which could limit its applicability. It consists mainly of BMI, weight loss, amount of food intake, age and disease severity that determine the risk of postoperative complications. The usefulness of this scale is well proven in surgery. Nonetheless, in urology, a good correlation between those variables could not be found. Only 1 research work from US confirmed the relationship between NRS 2002 and complications in patients after RC. ${ }^{6}$ In other research, malnutrition did not influence the duration of patients' stay in the urological ward. ${ }^{9}$ In a retrospective study of 905 patients, consisting of measuring BMI, weight loss and preoperative albumin level, "only preoperative albumin level was significantly associated with allcause mortality (...) within 90 days". ${ }^{8}$ In our study, the BMI indicator itself was much more reliable. As we can see from the correspondence analysis and dendrogram, undernutrition and obesity are associated with higher rate in ClavienDindo scale. There is no doubt that the "best patient" to be operated is the patient with normal body weight or little overweight, and in good nutritional condition. However, we have to face the problem of an increasing number of malnutrition and obesity patients in our society and find ways how to decrease the complication rates in those patients. Body mass index was also a better parameter than weight loss in a multicenter prospective cohort study of 2,258 patients who underwent major intra-abdominal cancer surgery; patients with a preoperative BMI $<18.5$ had greater than a 5 -fold increased risk of perioperative mortality. ${ }^{21}$ Even if in urology the correlation is not so strong, we should try to identify the nutritional status more precisely. It seems desirable to apply nutritional intervention, especially in wellidentified malnourished patients, and, as a result, decrease the number of postoperative complications. Nevertheless, today there are no studies evaluating the role of nutrition supplementation in patients with urinary bladder cancer. Therefore, larger-scale prospective studies are needed to determine what are the best markers for nutritional status and whether nutritional intervention helps to decrease the number of complications in nutritionally deficient patients undergoing RC. ${ }^{22}$ For the patients with BMI above 30, who have a higher occurrence of bladder cancer and also, what was observed in our study, higher postoperative complications rate after $\mathrm{RC}$, probably the best way to decrease postoperative complications will be motivating them to lose weight when their cancer is at the early stage (T1) and a radical cystectomy is a distant prospective. ${ }^{3}$ Decreasing the amount of inflammatory and angiogenic factors, which are produced in fat tissue, could help stop the development of cancer. As the latest research shows, obesity is a 3 times more frequent cause of death as compared to undernutrition. A dramatic increase of BMI in the population is terrifying, as almost $30 \%$ of cancers could be avoided if we managed to keep the BMI under 25. ${ }^{23}$ Therefore, the best solution is, in our case, keeping BMI between 18.5 and 30 to avoid potential problems. We have to be aware that both extremes in BMI will increase postoperative complications and we should try to apply more preventive treatment to decrease our patients' postoperative suffering.

The conducted study shows some limitations - complications were measured during hospitalization, which, in turn, does not exclude the general influence on higher mortality rate in longer time after operation; and some patients underwent neoadjuvant chemotherapy, which could be an interfering factor. Nevertheless, the study was multicenter. 


\section{Conclusions}

There is no relationship between malnutrition, measured in the NRS scale, and postoperative complications in cases of patients with urinary bladder cancer after radical cystectomy. Only 1 significant relationship between the nutrition states was observed, measured by BMI scale and the degree in Clavien-Dindo scale. BMI under 18.5 and over 30 increases postoperative complications.

Nutritional Risk Screening 2002 is based mostly on loss of weight and identifies patients who are in undernutrition state and at risk of undernutrition and who may improve their condition by nutritional intervention. Such defined malnutrition does not show any correlation with postoperative complications in patients undergoing $\mathrm{RC}$. In this case, qualitative malnutrition is more probable than the quantitative one. An evaluation tool should be sought to determine malnutrition status in patients with bladder cancer more precisely and more attention should be paid to qualitative malnutrition, since quantity is important when it involves a quality change. Greater precision in identifying patients in need of nutritional support would certainly help in achieving better long-term results. Multimodal pre-rehabilitation program before surgery appears to be a promising perspective for the future.

\section{References}

1. Jarosz M, Dzieniszewski J, Szczygieł B, et al. Nutritional status of patients in hospitals in Poland. Il. More thorough assessment of nutritional status of adult patients. Pol Merkur Lekarski. 2003;15:151-154.

2. Dzieniszewski J, Jarosz M, Szczygieł B, et al. Nutritional status of patients in hospitals in Poland. I. Screening of adult patients. Pol Merkur Lekarski. 2003;15:144-150.

3. Qin Q, Xu X, Wang X, Zheng XY. Obesity and risk of bladder cancer: A meta-analysis of cohort studies. Asian Pac J Cancer Prev. 2013;14(5):3117-3121.

4. Burrus MT, Werner BC, Yarboro SR. Obesity is associated with increased postoperative complications after operative management of tibial shaft fractures. Injury. 2016;47(2):465-470.

5. Stevens SM, O'Connell BP, Meyer TA. Obesity related complications in surgery. Curr Opin Otolaryngol Head Neck Surg. 2015;23(5):341-347.

6. Cerantola $Y$, Valerio $M$, Hubner $M$, Iglesias $K$, Vaucher $L$, Jichlinski P. Are patients at nutritional risk more prone to complications after major urological surgery? Prevention and management of complications following radical cystectomy for bladder cancer. $J$ Urol. 2013;190(6):2126-2132.

7. Roghmann F, Gockel M, Schmidt J, et al. Complications after ileal conduit: Urinary diversion-associated complications after radical cystectomy [in German]. Urologe A. 2015;54(4):533-541.

8. Munbauhal G, Drouin SJ, Mozer P, et al. Malnourishment in bladder cancer and the role of immunonutrition at the time of cystectomy: An overview for urologists. BJU Int. 2014;114(2):177-184.

9. Jensen BT, Laustsen $\mathrm{S}$, Petersen AK, et al. Preoperative risk factors related to bladder cancer rehabilitation: A registry study. Eur J Clin Nutr. 2013;67(9):917-921.

10. Herranz Amo F, Garcia Peris P, Jara Rascon J, et. al. Usefulness of total parenteral nutrition in radical surgery for bladder cancer [in Spanish]. Actas Urol Esp. 1991;15(5):429-436.

11. Cantiello F, Cicione A, Autorino R, et al. Metabolic syndrome, obesity, and radical cystectomy complications: A clavien classification system-based analysis. Clin Genitourin Cancer. 2014;12(5):384-393.

12. Shpata V, Prendushi X, Kreka M, Kola I, Kurti F, Ohri I. Malnutrition at the time of surgery affects negatively the clinical outcome of critically ill patients with gastrointestinal cancer. Med Arch. 2014;68(4):263-267.
13. Xu T, Zhu Z, Wang X, et al. Impact of body mass on recurrence and progression in Chinese patients with $\mathrm{Ta}, \mathrm{T} 1$ urothelial bladder cancer. Int Urol Nephrol. 2015;47(7):1135-1141.

14. Comploj E, West J, Mian M, et al. Comparison of complications from radical cystectomy between old-old versus oldest-old patients. Urol Int. 2015;94(1):25-30.

15. Carli F, Awasthi R, Gillis C, Kassouf W. Optimizing a frail elderly patient for radical cystectomy with a prehabilitation program. Can Urol Assoc J. 2014;(11-12):E884-E887.

16. Li C, Carli F, Lee L, et al. Impact of trimodal prehabilitation program on functional recovery after colorectal cancer surgery: A pilot study. Surg Endosc. 2013;27(4):1072-1082.

17. Chang SS, Cookson MS, Baumgartner RG, Wells N, Smith JA. Analysis of early complications after radical cystectomy: Results of a collaborative care pathway. J Urol. 2002;167(5):2012-2016.

18. Barbosa-Silva MC. Subjective and objective nutritional assessment methods: What do they really assess? Curr Opin Clin Nutr Metab Care. 2008;11(3):248-254.

19. Seres DS. Surrogate nutrition markers, malnutrition, and adequacy of nutrition support. Nutr Clin Pract. 2005;20(3):308-313.

20. Schiesser M, Kirchhoff P, Muller MK, Schäfer M, Clavien PA. The correlation of nutrition risk index, nutrition risk score, and bioimpedance analysis withpostoperative complications in patients under going gastrointestinal surgery. Surgery. 2009;145(5):519-526.

21. Kinn AC, Lantz B. Vitamin $B_{12}$ deficiency after irradiation for bladder carcinoma. J Urol. 1984;131(5):888-890.

22. Mullen JT, Davenport DL, Hutter MM, et al. Impact of body mass index on perioperative outcomes in patients undergoing major intraabdominal cancer surgery. Ann Surg Oncol. 2008;15(8):2164-2172.

23. Kiyama $T$, Witte MB, Thornton FJ, Barbul $A$. The route of nutrition support affects the early phase of wound healing. JPEN J Parenter Enteral Nutr. 1998;22(8):276-279.

24. Bhaskaran K, Douglas I, Forbes $H$, dos-Santos-Silva I, Leon DA, Smeeth L. Body-mass index and risk of 22 specific cancers: A population-based cohort study of 5.24 million UK adults. Lancet. 2014;384(9945):755-765. 